\title{
GLAST, THE GAMMA-RAY LARGE AREA SPACE TELESCOPE
}

\author{
ALESSANDRO DE ANGELIS \\ Università di Udine and INFN Trieste, via delle Scienze 208, I-33100 Udine (Italy)* \\ E-mail: deangelis@ud.infn.it
}

\begin{abstract}
GLAST, a detector for cosmic $\gamma$ rays in the range from $20 \mathrm{MeV}$ to $300 \mathrm{GeV}$, will be launched in space in 2005. Breakthroughs are expected in particular in the study of particle acceleration mechanisms in space and of gamma ray bursts, and maybe on the search for cold dark matter; but of course the most exciting discoveries could come from the unexpected.
\end{abstract}

\section{Introduction}

The exchange between laboratory physics and astrophysics has demonstrated through centuries of science to be at the origin of fundamental discoveries. GLASTL, a detector for cosmic $\gamma$ rays in the range from $20 \mathrm{MeV}$ to $300 \mathrm{GeV}$, is built around this idea: both the design of the instrument and the guidelines for science originate from a partnership of researchers from High Energy Physics (HEP) and High Energy Astrophysics (HEA). The collaboration involves the US (NASA in particular), France, Italy, Japan and Sweden.

The study of $\gamma$ rays is fundamental for our understanding of the universet: $\gamma$ rays probe the most energetic phenomena occurring in nature, and several signatures of new physics are associated with the emission of $\gamma$ rays. Photons can travel essentially undeflected and unabsorbed in space, and thus they point with excellent approximation to the source of their emission. Moreover, we have the technology for building high-performance $\gamma$ detectors. The design of GLAST, which will be better described in the next section, is rather typical for a modern HEP detector: essentially a silicon tracker followed by a calorimeter.

GLAST comes after the scientific success of the Energetic Gamma Ray Experiment Telescope (EGRET) instrument on the Compton Gamma Ray Observatory H. Launched in 1991, EGRET made the first complete survey of the sky above $30 \mathrm{MeV}$. EGRET increased the number of identified $\gamma$ sources producing a catalog which is a reference. Although EGRET answered many questions, it opened as many mysteries, which GLAST hopes to solve. GLAST maintains the same design philosophy as EGRET, but will improve substan-

\footnotetext{
*also at Instituto Superior Técnico, Lisboa.
}

University of Udine Report UDPHIR 00/02/AA, September 2000 


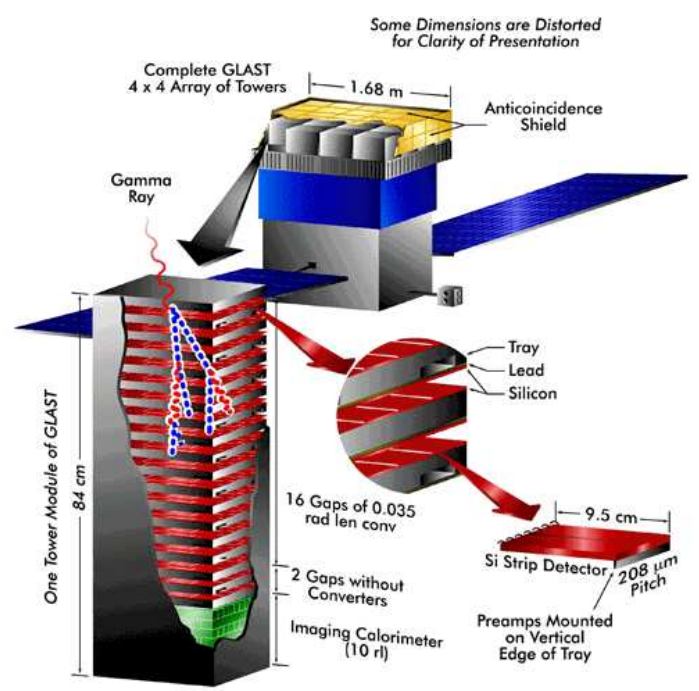

Figure 1. GLAST in detail.

tially the EGRET performance by using up-to-date technology from HEP.

GLAST will be sent in space in 2005; it should orbit at a height of $550 \mathrm{Km}$ and make a full sky coverage in $2-3$ orbits, sending data at a rate of $0.3 \mathrm{Mb} / \mathrm{s}$ (which could triplicate if the rapidly growing telecommunication technology will allow it). The detector is expected to take data for at least five years, with the possibility of extending the mission to ten years. The launch by a Delta 2 vector limits the weight of GLAST to around 4 tons and its size to $2 \times 2 \times 1 \mathrm{~m}^{3}$. The total power consumption is limited to $650 \mathrm{~W}$.

\section{The detector}

GLAST is an array of $4 \mathrm{x} 4$ identical towers (Figure 1), embedded in a plastic scintillator shield which serves as an anticoincidence trigger. Each tower, about $40 \times 40 \mathrm{~cm}^{2}$ in surface, comprises a tracker, a calorimeter and a data acquisition module.

The tracking detector consists of $18 x y$ planes of silicon strip detectors, with pitch of about $200 \mu \mathrm{m}$. On 16 of them a converter will increase the radiation length so to increase the probability of $\gamma$ conversion; the electronpositron pair coming from such conversion can be tracked to reconstruct with

University of Udine Report UDPHIR 00/02/AA, September 2000 


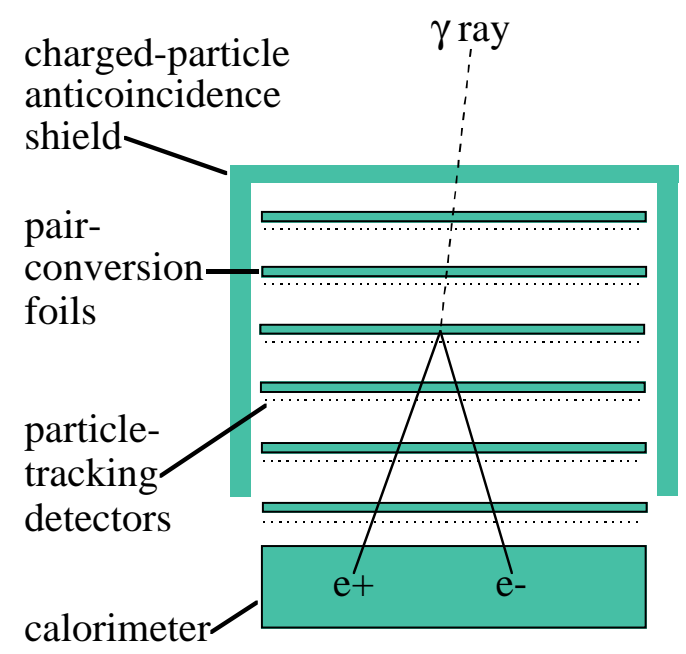

Figure 2. Principle of photon detection in GLAST.

accuracy the direction of the incoming photon. The choice of silicon guarantees high signal/noise ratio, radiation hardness and low power consumption. The system is mechanically stiffened by carbon walls; electronics is placed on the sides to minimize the gaps.

The calorimeter in each tower consists of 12 CsI bars in a hodoscopic arrangement, read out by double photodiodes; the total thickness is 10 radiation lengths. The hodoscopic geometry, with alternate $x$ and $y$ planes, allows fitting the shower profile, so to be able to compute the leakage and overcome the consequent deterioration of the energy resolution.

The setup consisting of a tracker and a calorimeter is a standard for the modern HEP experiments: the tracker detects the conversion and allows fitting the photon direction, while the calorimeter measures the total energy (Figure 2). The anticoincidence detector helps in suppressing the background from charged cosmic rays, which is five orders of magnitude larger than the $\gamma$ signal. The height/width ratio of $\approx 0.4$ guarantees a large field of view; the arrangement in 16 complete towers guarantees modularity.

As a result GLAST, built around the same design philosophy as EGRET, can increase substantially EGRET's sensitivity (Table 11).

GLAST is complementary to ground-based experiments, and is a key of the $\gamma$ astrophysics program for the new century (Figure 3).

University of Udine Report UDPHIR 00/02/AA, September 2000 


\begin{tabular}{lll}
\hline & EGRET & GLAST \\
\hline Energy range & $0.02-30 \mathrm{GeV}$ & $0.02-300 \mathrm{GeV}$ \\
Energy resolution $\Delta E / E$ & $10 \%(E>100 \mathrm{MeV})$ & $10 \%(E>100 \mathrm{MeV})$ \\
Single $\gamma$ angular resolution & $5.8^{\circ}(100 \mathrm{MeV})$ & $\simeq 3^{\circ}(100 \mathrm{MeV})$ \\
& & $\simeq 0.15^{\circ}(10 \mathrm{GeV})$ \\
Peak eff. area & $0.15 \mathrm{~m}^{2}$ & $1 \mathrm{~m}^{2}$ \\
Field of view & $0.5 \mathrm{sr}$ & $2.4 \mathrm{sr}$ \\
Time resolution & $100 \mu \mathrm{s}$ & $10 \mu \mathrm{s}$ \\
\hline
\end{tabular}

Table 1. Selected performance parameters for GLAST and EGRET.

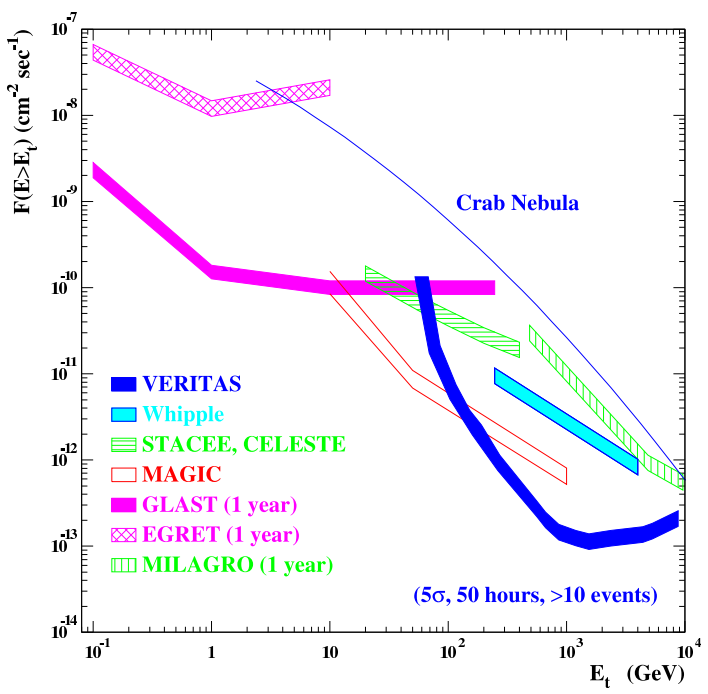

Figure 3. Sensitivity of present and future high energy $\gamma$ detectors.

\section{$3 \quad$ Key science objectives}

GLAST has a wide range of physics objectives, ranging from gamma astrophysics to fundamental physics. For a more complete review, see 1 and references therein. I try here just to give a short summary.

- Resolving the $\gamma$ ray sky: AGN, diffuse emission and unidentified sources EGRET has discovered $\simeq 80$ Active Galactic Nuclei (AGN) at $\gamma$ ray energies, and GLAST will discover several thousands; the $\gamma$ part of the 
spectrum could be dominant, and the variability in time of the sources will provide important information.

Most of the gamma-ray sources detected by EGRET remain unidentified; the accuracy of EGRET could allow identifying such sources.

Besides the AGNs, EGRET discovered a diffuse background radiation. Is it really diffuse, and thus produced at a very early epoch, or a flux from unresolved sources? GLAST will improve the angular resolution and the sensitivity to weak sources, possibly giving an answer.

- Acceleration mechanisms of Cosmic Rays (CR)

The signal of $\pi^{0}$ decaying into $\gamma \gamma$ could indicate the dominant role of the acceleration of nuclei. The angular resolution of GLAST will allow to possibly associate $\mathrm{CR}$ sources to supernova remnants.

- Gamma-Ray Bursts (GRB)

EGRET detected high energy afterglows (which can last for more than one hour) in 2-3 out of 6 GRBs observed; GLAST can provide measurements over a new energy range for 50-100 GRB/year. The notification of GRBs can be given by an on-board trigger (Gamma-Ray Burst Monitor) in a few seconds, and from earth in a few minutes.

In addition, recent quantum gravity models predict that the speed of photons depends on their energyt; this effect could cause delays of $\mathrm{O}(100 \mathrm{~ms})$ in the arrival time of photons from GRBs, and thus be detectable.

- Solar flares

GLAST could image these unexplained solar explosions, measuring the surface interested.

- Probing dark matter: WIMPs

If dark matter is made by Majorana particles weakly interacting and with thermic energies, the annihilation of such particles should result in narrow $\gamma$ lines. This is in particular true in SUSY, where the Lightest Supersymmetric Particle (LSP) can annihilate with itself producing $\gamma \gamma$ or $\mathrm{Z} \gamma$ pairs. GLAST can be sensitive to a non-negligible part of the space of the Minimal Supersymmetric Standard Model (MSSM) parameters for a LSP in the range from 30 to $100 \mathrm{GeV} / c$.

- Last but not least, the totally unexpected could come from the newly opened exploration regimes.

University of Udine Report UDPHIR 00/02/AA, September 2000 


\section{Status of GLAST}

The GLAST design has been extensively studied by means of simulations, and test beams are well on the way since three years to verify the expected performance.

In particular, the possibility of the calorimeter to reach a relative energy resolution $\Delta E / E \simeq 10 \%$ by fitting the shower profile has been successfully verified by dedicated tests in 1997 .

A full GLAST tower has been tested at SLAC in 1999/2000 with incident electron, photon and hadron beams. It has been verified in particular that a rejection factor of $10^{5}$ against hadrons is realistic, still keeping $80 \%$ of the photons.

The software for the simulation, the analysis and the distribution of data is being developed along the line of modern data handling technologies; simulation and reconstruction are well advanced and being used on the test data. A particular mention to the simulation: the detector project has been done with detailed simulations based on GISMOE, and now a simulation is being developed between Europe and Japan in close collaboration with the CERN Geant4 6 development team, which profits also of the interaction with ESA.

\section{$5 \quad$ Summary and conclusions}

GLAST will provide an important step forward in $\gamma$ astronomy, increasing the sensitivity of EGRET by one-two orders of magnitude.

The progress is in schedule for the launch in 2005; beam tests and software developement are well on the way.

We are confident that this partnership between HEP and HEA will be the source of new discoveries over a wide range of subjects.

\section{References}

1. See http://glast.gsfc.nasa.gov/ and references therein.

2. For a review, see for example C.M. Hoffman, C. Sinnis, P. Fleury and M.

Punch, Rev. Mod. Phys 71 (1999) 897.

3. See http://cossc.gsfc.nasa.gov/cossc/egret/and references therein.

4. G. Amelino-Camelia et al., Nature 393 (1998) 763.

5. T. Burnett and B. Atwood, http://glast.phys.washington.edu/ burnett/gismo/

6. http://wwwinfo.cern.ch/asd/geant4/geant4.html

University of Udine Report UDPHIR 00/02/AA, September 2000 